\title{
NOTCH1 PEST domain variants are responsive to standard of care treatments despite distinct transformative properties in a breast cancer model
}

\author{
Karen Cravero ${ }^{1, *}$, Morgan V. Pantone ${ }^{2, *}$, Dong Ho Shin ${ }^{2, *}$, Riley Bergman ${ }^{2}$, Rory \\ Cochran ${ }^{1}$, David Chu ${ }^{1}$, Daniel J. Zabransky ${ }^{1}$, Swathi Karthikeyan ${ }^{1}$, Ian G. Waters ${ }^{1}$, \\ Natasha Hunter $^{1}$, D. Marc Rosen ${ }^{1}$, Kelly Kyker-Snowman ${ }^{1}$, W. Brian Dalton ${ }^{1}$, Berry \\ Button $^{1}$, Dan Shinn ${ }^{1}$, Hong Yuen Wong ${ }^{2}$, Joshua Donaldson², Paula J. Hurley², \\ Sarah Croessmann ${ }^{2}$ and Ben Ho Park ${ }^{2}$ \\ ${ }^{1}$ The Sidney Kimmel Comprehensive Cancer Center, The Johns Hopkins University School of Medicine, Baltimore, MD, USA \\ ${ }^{2}$ Division of Hematology, Oncology, Department of Medicine, Vanderbilt University Medical Center and The Vanderbilt-Ingram \\ Cancer Center, Nashville, TN, USA \\ *These authors contributed equally to this work
}

Correspondence to: Ben Ho Park, email: ben.h.park@vumc.org

Keywords: NOTCH1; TNBC; breast cancer; PEST

Received: December 15, $2021 \quad$ Accepted: February 07, $2022 \quad$ Published: February 16, 2022

Copyright: ( $\odot 2022$ Cravero et al. This is an open access article distributed under the terms of the Creative Commons Attribution License (CC BY 3.0), which permits unrestricted use, distribution, and reproduction in any medium, provided the original author and source are credited.

\section{ABSTRACT}

Activating variants in the PEST region of NOTCH1 have been associated with aggressive phenotypes in human cancers, including triple-negative breast cancer (TNBC). Previous studies suggested that PEST domain variants in TNBC patients resulted in increased cell proliferation, invasiveness, and decreased overall survival. In this study, we assess the phenotypic transformation of activating NOTCH1 variants and their response to standard of care therapies. AAV-mediated gene targeting was used to isogenically incorporate 3 NOTCH1 variants, including a novel TNBC frameshift variant, in two non-tumorigenic breast epithelial cell lines, MCF10A and hTERT-IMEC. Two different variants at the NOTCH1 A2241 site (A2441fs and A2441T) both demonstrated increased transformative properties when compared to a non-transformative PEST domain variant (S2523L). These phenotypic changes include proliferation, migration, anchorage-independent growth, and MAPK pathway activation. In contrast to previous studies, activating NOTCH1 variants did not display sensitivity to a gamma secretase inhibitor (GSI) or resistance to chemotherapies. This study demonstrates distinct transformative phenotypes are specific to a given variant within NOTCH1 and these phenotypes do not correlate with sensitivities or resistance to chemotherapies or GSIs. Although previous studies have suggested NOTCH1 variants may be prognostic for TNBC, our study does not demonstrate prognostic ability of these variants and suggests further characterization would be required for clinical applications.

\section{INTRODUCTION}

Triple negative breast cancer (TNBC) is a subtype of breast cancer that accounts for $15-20 \%$ of all diagnosed patients. Traditionally, breast cancer is treated based on the expression of estrogen receptor (ER), progesterone receptor (PR), and/or human epidermal growth factor 2
(HER2) receptor using endocrine and HER2 targeting therapies, respectively. However, patients with TNBC lack expression of these receptors and are therefore limited to standard surgery, chemotherapy, and radiation for treatment options. More recently, immunotherapy has been approved for select patients with TNBC in both the metastatic and early-stage settings. Treatment efficacy is 
complicated by the heterogenous nature of TNBC. In 2011, Lehmann et al. demonstrated TNBC can be classified into distinct subtypes based on gene expression profiles and these molecular differences may dictate response to therapy [1]. These distinct molecular differences combined with the aggressive nature of TNBC have resulted in poor treatment options, increased rates of recurrence and metastases, and decreased overall survival [2-4]. Therefore, there is a pressing need to find alternative treatment options for patients with TNBC. In recent years, clinical studies for TNBC have begun focusing on new targeted therapies, such as poly (ADP-ribose) polymerase (PARP) inhibitors, anti-Trop2 antibody drug conjugates and immunotherapies, along with potential predictive markers for these therapies [5].

One potential target for novel therapies against TNBC is NOTCH1. NOTCH1 variants are found in many cancer types (cBioPortal) suggesting its pathogenic role in cancer growth, invasion, and metastasis [6-9]. Dysregulation of the Notch1 pathway has been frequently identified in different aggressive human cancers and has been shown to play an important role in cancer development [10-14]. Normal Notch signaling is carried out by four different single-pass transmembrane receptors and is essential for cell differentiation. The canonical activation of Notch1 signaling releases the Notch Intracellular Domain (NICD), which translocates to the nucleus upon activation. The $\gamma$-secretase (GS) complex is required for cleavage and activation of all four Notch receptors [15]. Aberrant activation of the Notch1 pathway is often the result of PEST domain variants that lead to stabilization of NICD and constitutive activation of Notch1 signaling [16, 17]. Several studies have demonstrated specifically frameshift and truncating PEST domain variants increase the stability and half-life of NICD [18-21].

Notch1 signaling is activated at a significantly higher rate in TNBC compared to other subtypes of breast cancer [18, 22, 23]. Among TNBC patients, Notch1 receptor variants range from missense to frameshift variants and cluster within the PEST domain region (cBioPortal) [24]. Furthermore, TNBC patients with increased Notch1 expression have demonstrated increased aggressive phenotypes and lower median overall survival [25]. In more recent years, the high correlation of aberrant Notch1 signaling and TNBC has gained interest as a potential target for new therapies. In 2015, Wang et al. demonstrated $\gamma$-secretase inhibitors (GSI) could disrupt Notch1 activation in patient-derived xenografts with PEST domain variants [18]. In the following years, GSIs have been explored in phase I and II clinical trials for breast cancer, pancreatic cancer, and metastatic melanoma [26-28]. While there is still no proven clinical benefit in breast cancer patients, this avenue of therapy may provide a targeted-treatment opportunity for TNBC patients. In our current study we generated and characterized a panel of isogenically modified NOTCH1 cell lines to characterize the transformative potential of these variants in nontumorigenic breast epithelial cells and tested the predictive value of these variants to GSIs and current standard of care chemotherapies for TNBC.

\section{RESULTS}

\section{NOTCH1 PEST domain variants in non- tumorigenic human breast epithelial cell lines result in NICD Notch1 activation}

Analysis of three publicly available tumorassociated variant databases (TSGene [29], COSMIC [30], and cBioPortal [24]) identified the NOTCH1 A2441 site as a commonly mutated codon in breast as well as many other cancers (salivary, adrenal, T-ALL, etc). To study the transformative properties of NOTCH1 variants in nontumorigenic breast epithelial cells, a small cohort of variants were selected for gene targeting. Among the numerous A2441 variants, a frameshift insertion variant (A2441Efs*39, abbreviated A2441fs) and missense variant (A2441T) were selected. In addition to these variants, a PEST domain variant (S2523L) located downstream of the A2441 site was selected to compare transformative properties (Figure 1A). AAV-mediated gene targeting was used to isogenically incorporate the NOTCH1 variants into two non-tumorigenic breast epithelial cell lines (MCF10A and hTERT-IMEC). In addition to the NOTCH1 variants, a targeted wildtype (TWT), which underwent the same gene targeting mechanism with a wildtype vector, was generated for both parental cell lines to act as a control. Schematic representation of AAV-mediated gene targeting is shown in (Figure 1B). PCR and Sanger sequencing were done to confirm a single allelic copy of the desired variant in each clone (Figure 1C). As mentioned previously, frameshift and truncating variants in NOTCH1 result in an extended half-life for the cleaved NICD protein and constitutively active Notch1 pathway. To confirm the novel A2441fs variant had increased cleaved NICD, immunoblot assays for Notch1, Notch1 transmembrane (NTM), and Notch1 intracellular domain (NICD) were performed for both the MCF10A and hTERT-IMEC panels. In both panels, the novel A2441fs variant had a second band present for the NICD immunoblot, representing the higher levels of cleaved Notch1 (Figure 1D).

\section{NOTCH1 A2441 variants confer growth factor independence and increased MAPK activity}

Increased proliferative signaling is a traditional hallmark of cancer and arguably the most fundamental trait of cancer cells [31]. Increased proliferation can be the result of a variety of processes including, but not limited to, increased signaling, loss of ligand dependence, production of growth ligands, or decreased thresholds for response 
[31]. To determine if $\mathrm{NOTCH1}$ variants caused increased proliferation rates in non-tumorigenic cell lines, the MCF10A and hTERT-IMEC cell line panels were grown in standard growth factor supplemented media. There was no significant difference between the $\mathrm{NOTCH} 1$ variants and their controls for either the MCF10A or hTERT-IMEC panels (Figure 2A, Supplementary Figure 1A). MCF10A and hTERT-IMEC cells require epidermal growth factor (EGF) supplementation for normal proliferation. EGF is the activating ligand of EGFR, a receptor tyrosine

A



C

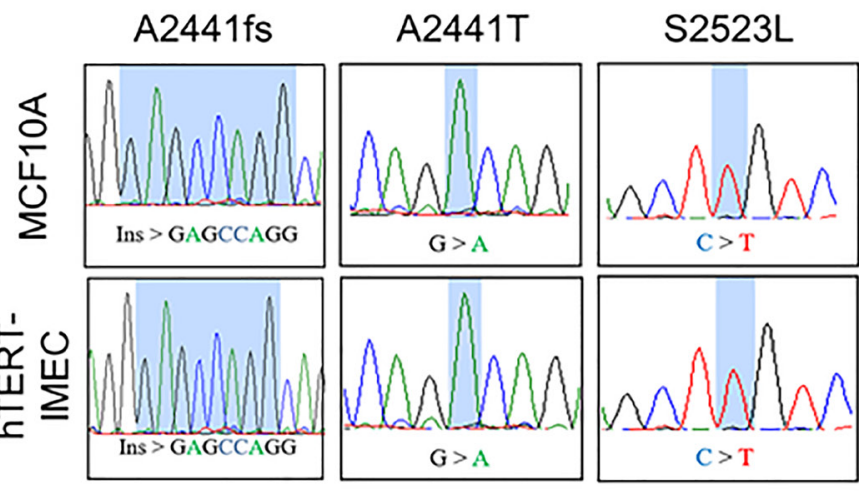

D

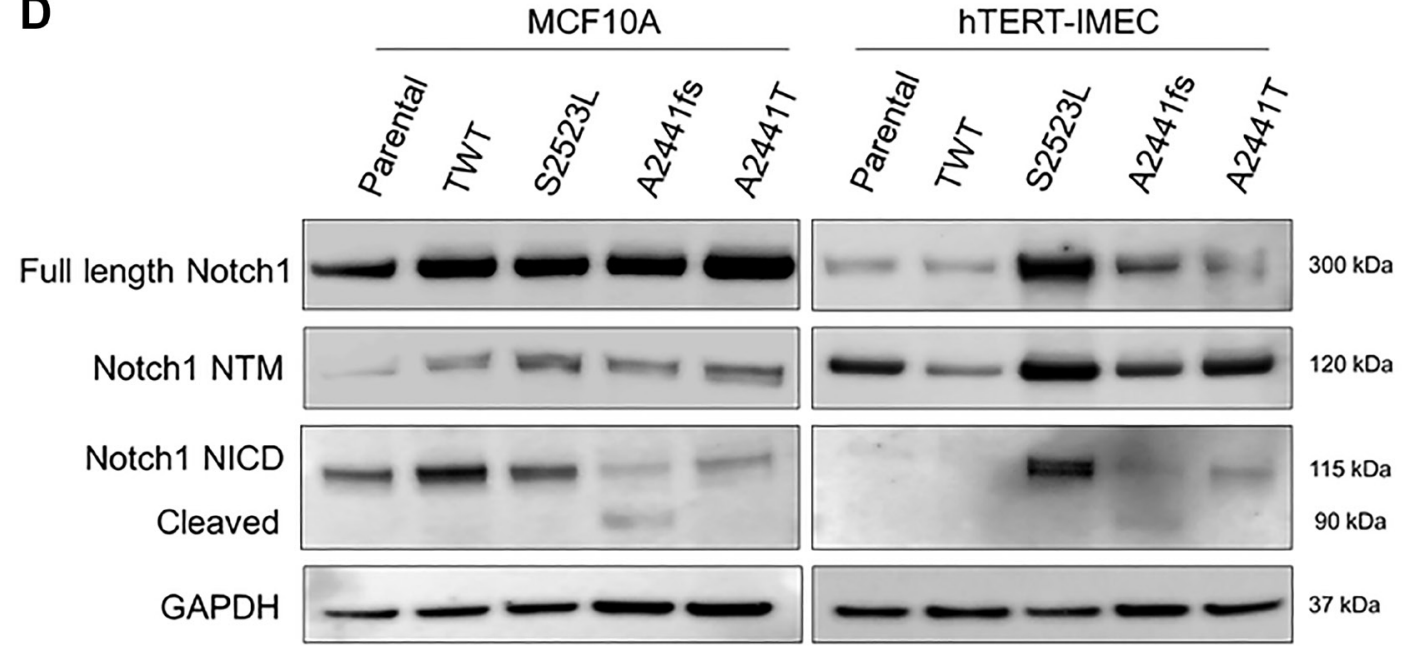

MCF10A

hTERT-IMEC

Figure 1: NOTCH1 isogenic cell panel in nontumorigenic breast epithelial cells. (A) Representative NOTCH1 variants included in the isogenic panel. A2441fs was identified in a tumor board and A2441T and S2523L were identified via cBioPortal. (B) Schematic of rAAV-mediated gene targeting of variants in exon 34 of NOTCH1. rAAV transduction leads to locus-specific targeting via homologous recombination of the $5^{\prime}$ and $3^{\prime}$ homology arms (HA). After neomycin selection, the isolated clone is subjected to Cre recombinase to excise the neomycin cassette $\left(\mathrm{Neo}^{\mathrm{R}}\right)$, resulting in a LoxP site. (C) Sanger sequence confirmation of genomic alterations in the NOTCH1-PEST cohort for both the MCF10A and hTERT-IMEC panel. (D) Immunoblot analysis for Notch1, Notch1 transmembrane (NTM), and Notch1 intracellular domain (NICD) for both the MCF10A and hTERT-IMEC panels. Cleaved version of Notch1 NICD for A2441fs is visualized by the NICD antibody. 
kinase, that is often dysregulated in many malignancies. Removal of EGF from non-tumorigenic cells results in G1 arrest. Ligand-independent activation of the EGFR pathway has been associated with increased malignant potential [32]. To determine if NOTCH1 variants impart a ligand-independent proliferative advantage, growth assays were carried out in the absence of EGF. Our results demonstrate within both NOTCH1 panels, clones with variants at the A2441 site (A2441fs, A2441T) exhibited EGF-independent growth but the S2523L variant did not (Figure 2B, Supplementary Figure 1B). Clonogenic growth assays in the absence of EGF were carried out over 14 days and confirmed EGF-independent growth for both NOTCH1 A2441 variants (Figure 2C, Supplementary Figure 1C).

Constitutive activation of EGFR is often the result of somatic variants, gene amplification, and/or signaling in oncogenes and leads to upregulation of the MAPK and PI3K signaling pathways [33]. To determine if either of these pathways were upregulated in the NOTCH1 cell line panels, immunoblot analyses were carried out in the absence of EGF. For both variants at the A2441 site but not the S2523L variant, there was a significant increase in phospho-MEK and phospho-ERK, two downstream effectors of the MAPK pathway (Figure 2D, Supplementary Figure 1D). Interestingly, the A2441 variants did not have elevated phospho-AKT compared with controls, suggesting the NOTCH1 A2441 variants activate the MAPK pathway but not the PI3K pathway.

\section{EGF-independent Notch1 variants confer invasive growth phenotype and dysregulated 3D morphology}

Anchorage independent growth in soft agar is a characteristic of cancer-associated variants and best correlates with in vivo tumorigenicity [34]. MCF10A cells cannot form colonies in semi-solid media or tumors in mice, however previous studies have demonstrated that aggressive genetic variants can lead to invasive colony formation in soft agar and in vivo tumor formation in athymic nude mice [35, 36]. In physiological doses of EGF the NOTCH1 A2441 variants formed large, proliferative colonies, while the S2523L variants appeared to quiesce (Figure 3A). Interestingly, the A2441 variants were also capable of forming colonies in the absence of EGF (Supplementary Figure 2A and 2B). To determine if these variants affected the morphology of three-dimensional growth, acini formation assays were carried out in Matrigel. Normal MCF10A cells form uniform, hollow, acinar structures that retain important characteristic of glandular epithelium such as low proliferation and stable uniform structure [37]. In the absence of EGF, both parental cell lines and the S2523L variant were unable to form acini. However, both NOTCH1 A2441 variants formed a significant number of colonies (Figure
3B, Supplementary Figure 2C-2E). Interestingly, in the presence of physiological doses of EGF, the A2441T variant demonstrated morphological changes in both the MCF10A and hTERT-IMEC cell lines, including protrusions and bridging suggesting loss of structural integrity and an increased transformative phenotype (Figure 3C). The ability to bridge between structures in semisolid media also suggests increased invasive and migratory potential.

\section{EGF-independent Notch1 variant cells have increased migratory potential in vitro}

Migratory capacity is associated with anchorage independent growth and increased transformative phenotypes in transformed cells. Previous studies have demonstrated Notch1 overexpression leads to increased migratory potential in vitro and may indicate increased metastatic potential $[38,39]$. To determine if the NOTCH1 A2441 variants demonstrated increased migratory capacity, a scratch wound assay under physiological EGF conditions was carried out in the MCF10A and hTERTIMEC cell line panels. In the MCF10A panel, both A2441 variants demonstrated significant wound closure when compared to the WT controls and the non-phenotypic S2523L variant, (Figure 3D and 3E). Interestingly, there was no significant difference between variants and controls in the hTERT-IMEC cell line panel (Figure $3 F$, Supplementary Figure 3). These results suggest that variants in the A2441 variants can confer an increase in migratory potential, but that cell line context also plays a role in mediating this phenotype. Taken together with the ability to grow in semi solid media, these data suggest variants at the NOTCH1 A2441 site may confer increased metastatic potential.

\section{The NOTCH1 A2441T variant alters gene expression in cancer pathway genes}

Notch signaling plays a fundamental role in cell differentiation and proliferation and activation of Notch signaling interacts with numerous oncogenic pathways [14]. To determine if variants at the A2441 site result in differences in gene expression, a microarray targeting over 500,000 transcripts including coding, non-coding genes, as well as exons, and splice variants $\left(\right.$ Clariom $^{\mathrm{TM}} \mathrm{D}$ human assay) was used to compare the $\mathrm{A} 2441 \mathrm{~T}$ variant to the MCF10A TWT. The A2441T site was selected due to the increased transformative properties in $3 \mathrm{D}$ medium. It is important to note the microarray was carried out in the presence of EGF to allow for active proliferation of both the variant and the TWT control. The microarray analysis identified 3106 differentially expressed transcripts in the A2441T cells. Out of the total number of dysregulated genes, 1805 genes were up-regulated and 1301 were down-regulated (Figure 4A and 4B). Raw expression 
values $(\log 2)$ from three independent runs for each cell line were grouped using a hierarchical clustering algorithm and presented as a heat map (Figure 4C). The clustering confirmed the A2441T mutant cell lines demonstrated a distinct gene expression profile when compared to the TWT control. Among the 3106 differentially expressed genes, the most abundantly over and under expressed genes in A2441T were analyzed. Among the 20 genes with

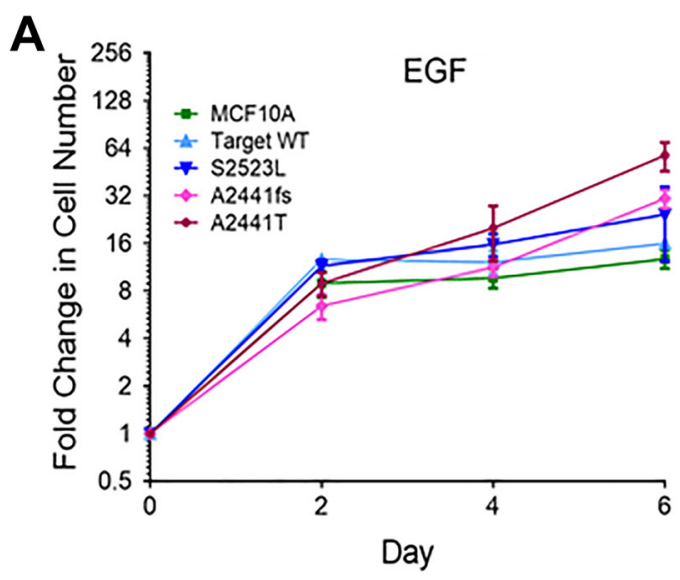

C

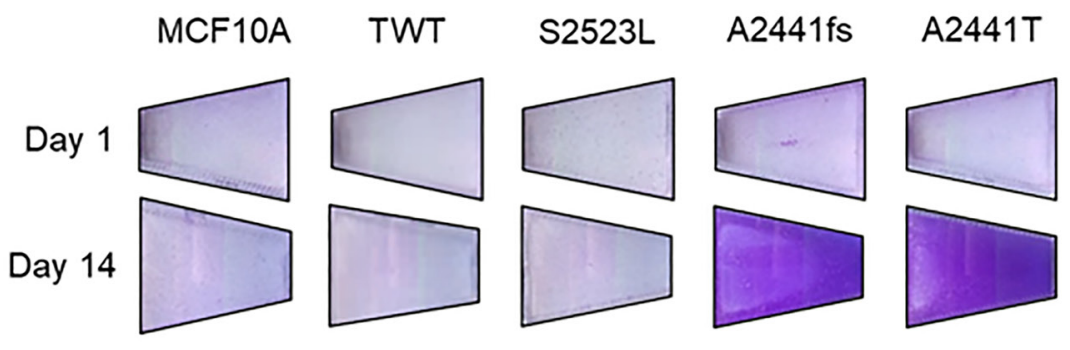

D

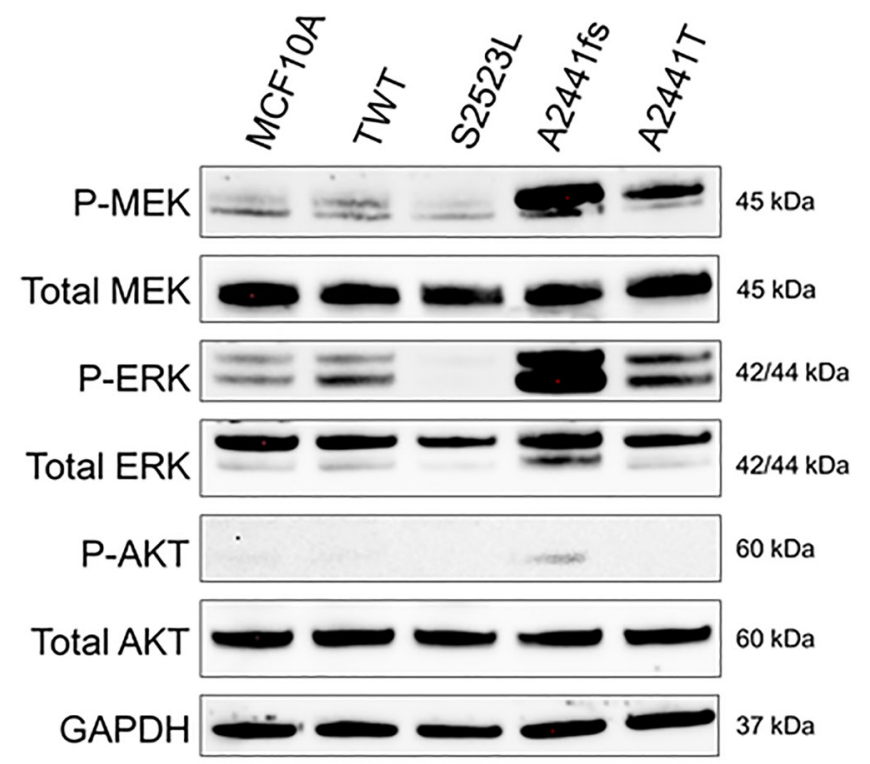

Figure 2: Some NOTCH1 PEST domain variants lead to growth-factor independent proliferation. (A) Relative mean growth of MCF10A NOTCH1 variant panel in the presence of physiological $0.2 \mathrm{ng} / \mathrm{mL}$ epidermal growth factor (EGF). Data are representative of the mean $\pm \operatorname{SEM}(n \geq 3)$. (B) Relative mean growth of MCF10A NOTCH1 variant panel in the absence of EGF. Data are representative of the mean $\pm \operatorname{SEM}\left(n \geq 3,{ }^{* * *} P \leq 0.001,2\right.$-way ANOVA followed by Bonferroni multiple comparison test). (C) Representative images of EGF independent growth in the MCF10A NOTCH1 panel stained with crystal violet on day 1 and day 14. (D) Immunoblot analysis of the MCF10A panel in the absence of EGF. 


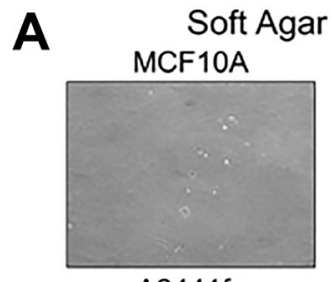

A2441fs

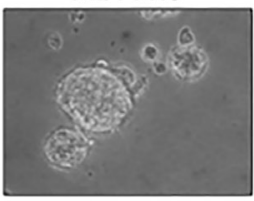

B

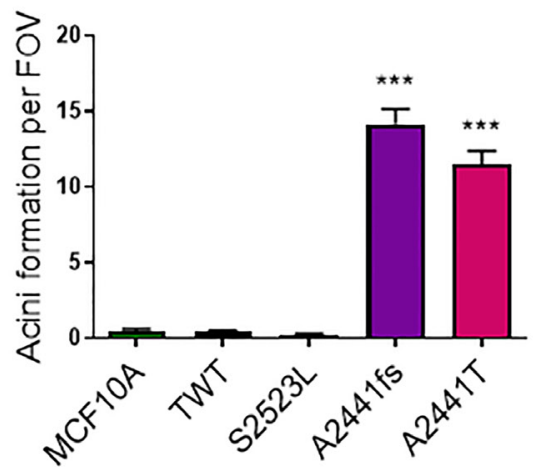

C

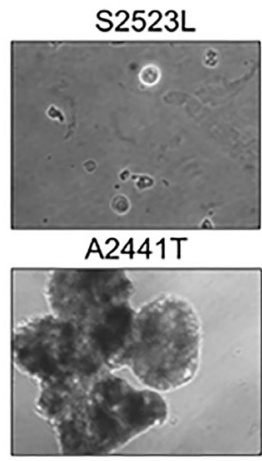

D

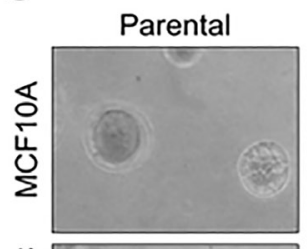

Acini Formation


$\mathbf{E}$

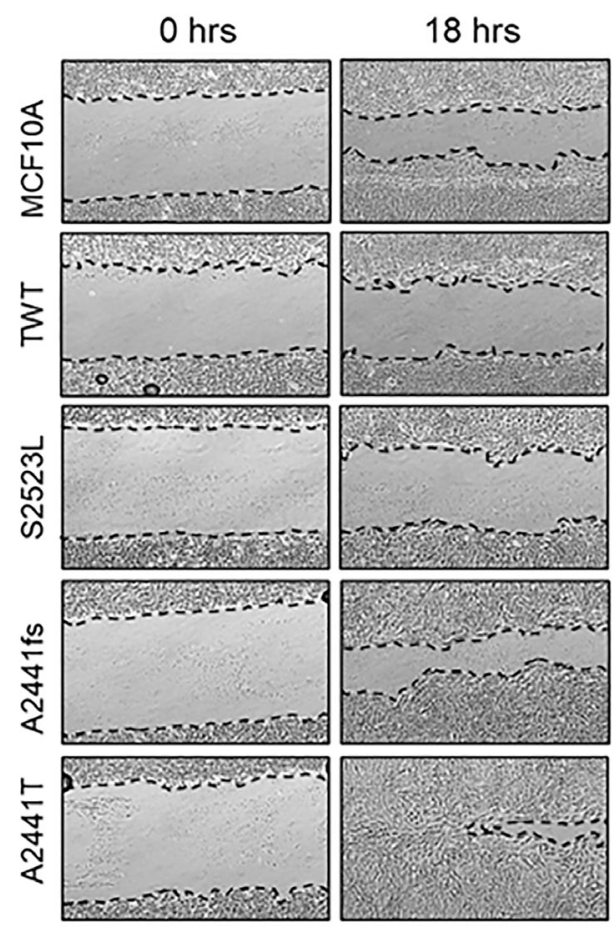

$\mathbf{F}$



$18 \mathrm{hrs}$

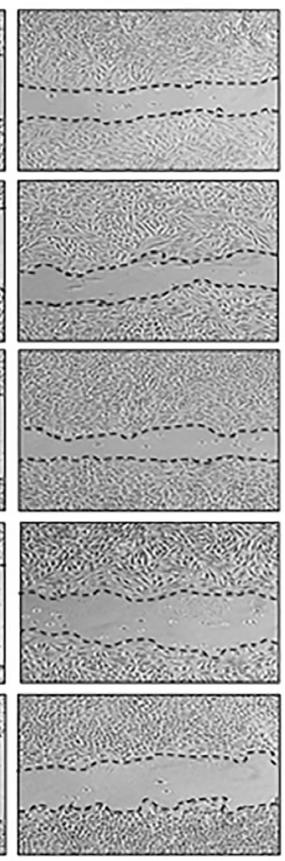

Figure 3: EGF-independent NOTCH1 variants demonstrate increased transformative phenotypes. (A) Representative colony formation in semisolid medium cultured for 3 weeks. MCF10A cells were seeded in $0.8 \%$ soft agar plate at low density in physiologic doses of EGF $(0.2 \mathrm{ng} / \mathrm{mL})$. Magnification $=100 \times$. (B) Matrigel acinar formation assay. Quantification of acini per field of view (FOV). ( $n$ $\geq 3,{ }^{* * *} P \leq 0.0012$-way ANOVA followed by Bonferroni multiple comparison test). (C) Representative images of irregular morphology in the A2441T variant panel when cultured in Matrigel in the physiologic doses of EGF. Magnification $=400 \times$. (D) Quantification of wound closure assay. Percentage of wound closure was measured at time of scratch (time 0 ) and after 18 hours. Data are representative of the mean $\pm \operatorname{SEM}\left(n \geq 3,{ }^{* *} P \leq 0.01,{ }^{* * *} P \leq 0.0012\right.$-way ANOVA followed by Bonferroni multiple comparison test). Representative images of wound closure assay of (E). MCF10A (F). hTERT-IMEC NOTCH1 variant panel. 
the highest and lowest expression, $75 \%$ of genes have been implicated in carcinogenesis (blue and pink bars, Figure 4D) and 30\% of genes have been linked to breast cancer (pink bars, Figure 4D) [40]. Furthermore, we queried for genes commonly altered in TNBC [41] and found several were dysregulated in the A2441T variant (Supplementary Figure 4). There were no substantive differences in terms of MAPK signaling between A2441T knock in cells and wild type cells when grown in the presence of physiological doses of EGF. Taken together, these data

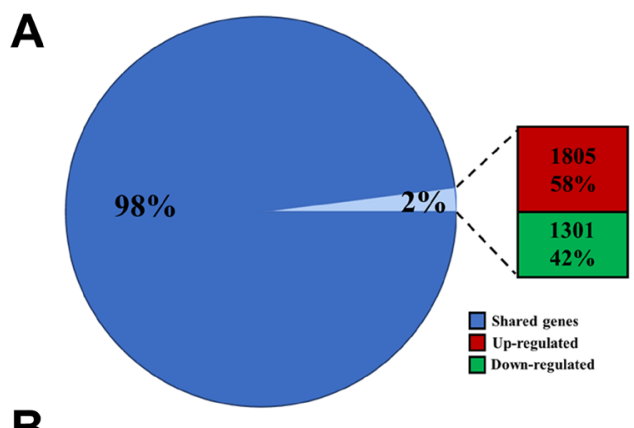

B


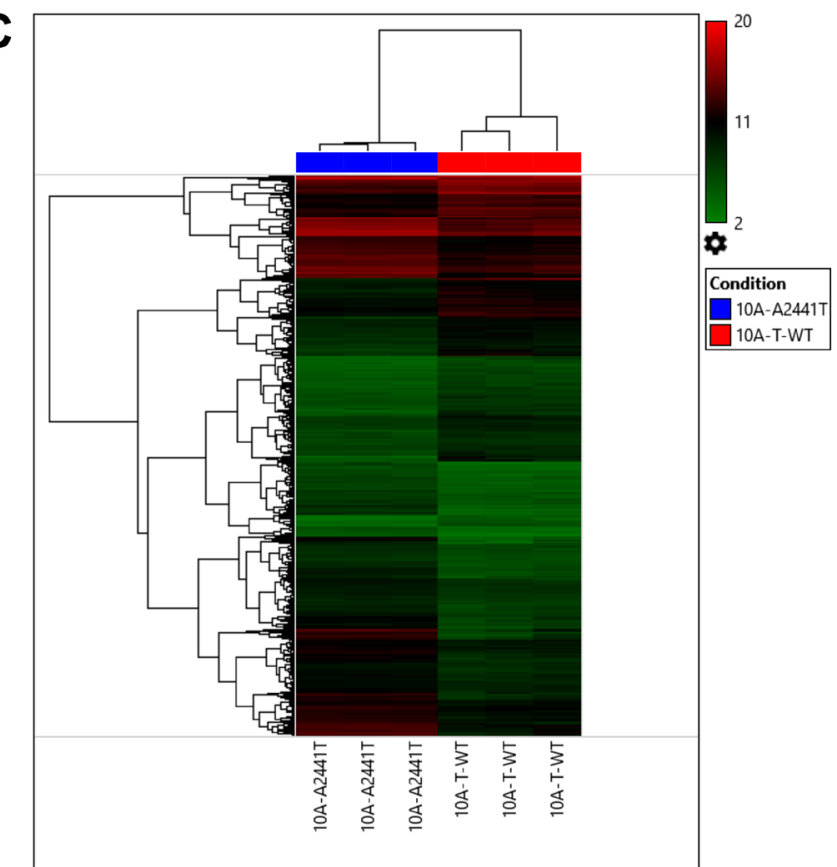

Figure 4: NOTCH1 PEST variants confer gene expression changes in nontumorigenic cell lines. MCF10A A2441T variant and TWT cells were subjected to a microarray analysis with 500,000 transcripts including coding, noncoding, and splice variants. (A) Percentage of differentially expressed transcripts. (B) Volcano plot of 3106 differentially expressed transcripts (red, upregulated; green, downregulated) between A2441T and TWT. (C) Raw expression values (log2) for triplicate runs in each cell lines were grouped using a hierarchical clustering algorithm and are presented as a heat map (red, upregulated; green, downregulated). (D) The 20 genes with the highest, and 20 genes with the lowest expression in A2441T cells based on fold change. Based on a literature review, genes associated with cancer shown in blue and genes associated with breast cancer shown in red. 
suggest NOTCH1 A2441 variants can significantly alter gene expression in non-tumorigenic breast epithelial cells.

\section{NOTCH1 A2441 variants do not confer differential responses to therapies}

Sequencing efforts to identify potential therapeutic targets or biomarkers for TNBC has led to the distinct association of NOTCH1 variants with TNBC. Recently, in vitro studies have shown GSIs preferentially target NOTCH1 alterations and may offer a new therapeutic option for patients with TNBC [18, 42, 43]. Furthermore, in 2017 a phase I clinical trial determined the GSI nirogacestat in combination with chemotherapy was well tolerated in patients with metastatic TNBC [44]. To determine if variants at the A2441 site were specifically susceptible to GSIs or resistant to standard of care chemotherapies, IC50s for each cell line were determined for nirogacestat as well as 5 common chemotherapies. In both the MCF10A and hTERT-IMEC panels there was no observable difference in IC50s for the NOTCH1 variants when compared to controls (Figure 5A, Supplementary Figures 5 and 6). For nirogacestat, IC50s for MCF10As and modified variants ranged between 8.6 and $10.2 \mu \mathrm{M}$. These ranges are considered to be on the high end of concentrations based on previous publications [15]. This may, in part, be due to the independent establishment of parental MCF10As without aberrant pathway activation. Furthermore, previous studies have demonstrated IC50s for breast cancer cell line models are significantly higher than in vitro concentrations for other cancer cell types [45]. Therefore, to confirm these findings in a clinical setting, we utilized the publicly available METABRIC data set via cBioPortal to determine if NOTCH1 variants conferred a differential response to chemotherapies. Selection criteria for the comparison included patients with TNBC who received at least one chemotherapy ( $n$ $=149)$. When comparing patients with $\mathrm{NOTCHI}$ variants $(n=11)$ to patients without NOTCH1 variants $(n=138)$, there was no significant difference in overall survival (Figure 5B) or progression free survival (Figure 5C). Although these sample sizes are small, they confirm our in vitro observations and suggest that despite the phenotypic changes due to the NOTCH1 A2441 variants, they do not provide predictive value for response to chemotherapies or nirogacestat.

\section{DISCUSSION}

This study investigated a novel NOTCH1 PEST domain frameshift variant as well as two previously reported variants to determine cancerous phenotypes and whether these variants could serve as predictive markers for therapeutic response in TNBC. TNBC is a particularly recalcitrant disease with limited treatment options. In recent years, there has been an increasing interest in identifying molecular targets that can be utilized as potential therapeutic targets or as prognostic biomarkers [41]. These efforts have led to the identification of Notch1 as a key player in breast carcinogenesis. However, clinical studies examining the relationship between Notch1 expression and clinical outcome are inconsistent and the prognostic value of Notch1 expression remains unclear [46]. Analysis of The Cancer Genome Atlas (TCGA) database found that activated Notch1 is reportedly mutated in approximately $13 \%$ of TNBCs, is strongly enriched in the basal subtype, and is positively correlated with breast cancer progression [18]. Furthermore, studies found that activating variants in the NOTCH1 PEST domain receptor are targetable oncogenic drivers in TNBC xenografts and are selectively responsive to GSIs [18]. However, due to the number and complexity of NOTCH1 variants across breast cancer compared to traditional hotspot variants, classification of $\mathrm{NOTCH} 1$ variants remains understudied.

Identification of transformative phenotypes within our NOTCH1 panel revealed that both variants at the A2441 site exhibited increased MAPK pathway activation, growth factor and anchorage independent growth, and increased migratory potential. Previous studies have demonstrated that frameshift variants in the PEST domain result in increased NICD and consequently, more aggressive phenotypes. However, the A2441T variant demonstrated increased transformation in semisolid medium (Figure 3C) when compared to a frameshift at the same site. Additionally, despite also being a PEST domain variant, the S2523L variant did not exhibit any transformative properties. Taken together, these data suggest that the oncogenic potential of NOTCH1 PEST domain variants depends on both variant type and amino acid location. Furthermore, previous studies have demonstrated the oncogenic potential of NOTCH1 variants is heavily dependent on the type of tumor, with some cancers suggesting NOTCH1 is an oncogene and others more consistent with a tumor suppressor gene. Our own study also shows that some phenotypes are cell type specific (increase migratory potential). The scratch wound assay showed increased closure with A2441T variants only in the MCF10A cell line but not in hTERT-IMECs. These results suggest other factors must be present or absent in hTERT-IMECs beyond the A2441T mutation and that the mutation is necessary but not sufficient to impart this phenotype. These two cell lines were derived from distinct persons, and established in different ways, with MCF10A being spontaneously immortalized while hTERT-IMECs were immortalized with hTERT overexpression. Therefore, it is not surprising that some phenotypes would be unique to one cell line. Indeed, we and others have shown in past studies differences in phenotypes and gene expression between these two cell lines [35, 47, 48]. Variability between NOTCH1 variants as well as genomic background indicates a need for expansive classification before they can be used as reliable prognostic and/or predictive markers. 
Clinical evidence has suggested NOTCH1 variants are linked to chemotherapy resistance and emerging functional studies have suggested that Notch inhibitors, including GSIs, can effectively target NOTCH1 variants $[18,49]$. However, within our cell line panels, regardless of oncogenic phenotypes, there was no significant difference in response to GSIs or chemotherapies when compared to controls. This suggests that despite Notch pathway activation, NOTCH1 variants are not useful predictive biomarker for treatment response and should currently not be considered when determining individual treatment courses. Despite evidence suggesting treatment resistant breast cancers can be re-sensitized by Notch inhibitors, our data provides strong rationale that further investigation is required [50]. The high variability among NOTCH1 variants and the expansive number across the gene make it difficult to attribute therapeutic sensitivity to the presence of any single NOTCH1 variant. This is emphasized by the lack of differential response across our panels to GSI and chemotherapies, despite demonstrating distinct phenotypes. The isogenic, non-tumorigenic background of our panels allows us to accurately assess the response to therapies for a specifically incorporated variant. Furthermore, the variants are expressed under the
A
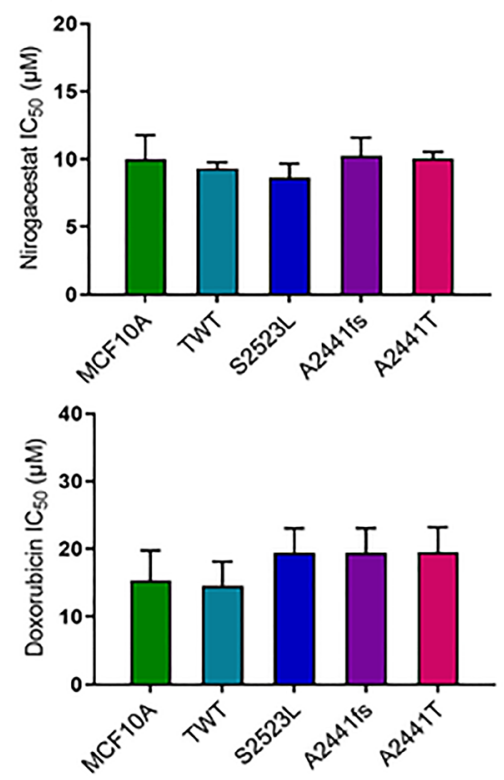

B

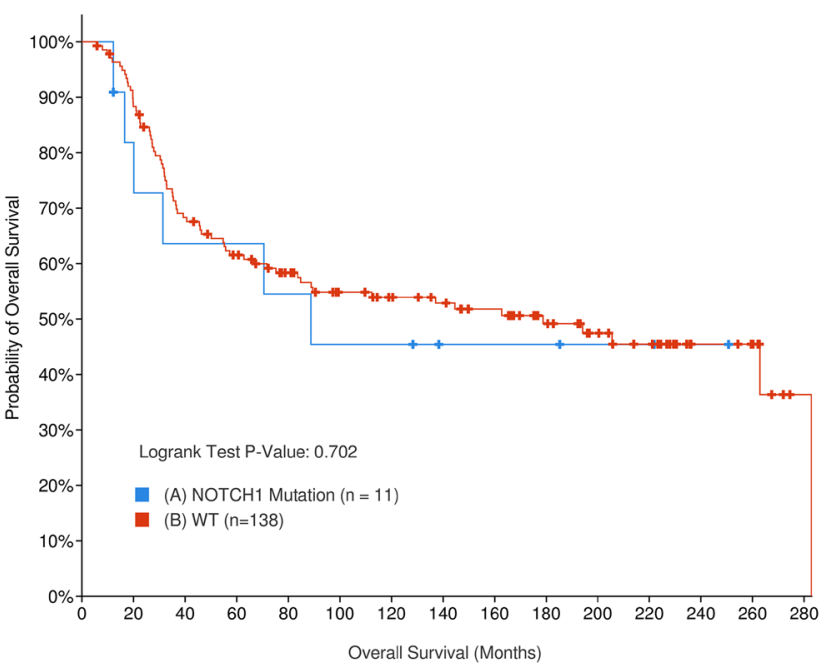

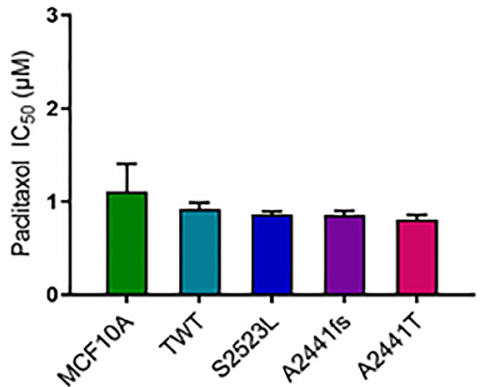


C



Figure 5: NOTCH1 PEST variants do not demonstrate differential response to TNBC standard of care therapies. (A) Cell counts of the NOTCH1 variant panel were used to determine the IC50s in 6 different standard of care therapies for TNBC (Nirogacestat, Paclitaxel, Cisplatin, Doxorubicin, Gemcitabine, and 5-FU). Data are representative of the mean $\pm \operatorname{SEM}(n \geq 3$, ns). (B) Overall survival and (C). progression free survival in TNBC patients treated with chemotherapy with (blue) and without (red) NOTCH1 variants. METABRIC cohort data set was analyzed via cBioPortal. 
endogenous promoter to provide a model representative of how the variant behaves in a patient's tumor. Future studies involving meticulous characterization of an expansive panel of NOTCH1 variants in a similar model may provide mechanistic insight and predictive and/ or prognostic value that is both variant type and site dependent.

\section{MATERIALS AND METHODS}

\section{Cell culture}

MCF10A cell lines were maintained in DMEM:F12 (Invitrogen) supplemented with 5\% horse serum (HS, Life Technologies), $20 \mathrm{ng} / \mathrm{mL}$ epidermal growth factor (EGF, Sigma), $0.5 \mu \mathrm{g} / \mathrm{mL}$ hydrocortisone (Sigma), $10 \mu \mathrm{g} / \mathrm{mL}$ insulin (Life Technologies), 1\% penicillin-streptomycin (Life Technologies), and $0.1 \mu \mathrm{g} / \mathrm{mL}$ cholera toxin (Sigma). hTERT-IMEC cell lines were maintained in DMEM:F12 supplemented media supplemented with $1 \%$ charcoal dextran stripped FBS (CD, Life Technologies) in place of horse serum. Parental cell lines were authenticated via short tandem repeat profiling analysis at the Johns Hopkins Genetic Resources Core Facility.

\section{Gene targeting and generation of $\mathrm{NOTCH1}$ variant cell lines}

Gene targeting of Notch1 in MCF-10A and hTERTIMEC cells was carried out using recombinant AAV vectors as previously described $[51,52]$. Briefly, targeting vectors were designed by site-directed mutagenesis through overlap extension PCR onto a parental AAV plasmid backbone. Viral vectors were packaged using HEK-293T cells and resulting virus was transduced into targeted cell lines. Neomycin selection and PCR screening were used to select cells for homologous integration of targeting vectors via our previously described PCR-based screening method [53]. Identified colonies were singlecell diluted and selection cassettes were removed using Cre recombinase. Primer sequences for gene targeting and screening can be found in Supplementary Table 1.

\section{Cell proliferation assay}

Cells were seeded in triplicate at $3 \times 10^{4}$ per well and serum-starved for 24 hours before the assay media was added. Assay media consists of DMEM:F12 media containing $1 \% \mathrm{CD}$, hydrocortisone, cholera toxin, insulin and varying levels of EGF as indicated (no EGF or 0.2 $\mathrm{ng} / \mathrm{mL}$ EGF). Medium was changed every three days and cells were counted using a Beckman Coulter counter. For crystal violet stains, cells were plated at the same density in a T25 flask, media was changed every three days, and cells were fixed and stained with 3.7\% formaldehyde containing $0.2 \%$ crystal violet (Sigma).

\section{Colony formation assay in semisolid medium}

Cells were plated at $3 \times 10^{3}$ cells onto a $0.6 \%$ agarose layer with assay medium in a 6-well plate as previously described [54]. $0.4 \%$ agarose layer with assay medium was placed on top and changed weekly. Photographs were taken with a Nikon SMZ 1500 stereoscopic zoom microscope.

\section{Acinar morphogenesis assay}

Morphogenesis assays were conducted in the growth factor reduced Matrigel (BD Biosciences) as previously described [54]. Cells were seeded in 8-well chamber slides containing a solid base layer of growth factor reduced Matrigel (BD Biosciences). Photographs were taken with a Nikon SMZ 1500 stereoscopic zoom microscope.

\section{Scratch wound healing assay}

Cells were plated in six-well plates and grown under physiological growth conditions $(0.2 \mathrm{ng} / \mathrm{mL}$ EGF) to near confluent monolayers. Scratch wounds were introduced in a cross pattern with a $200-\mu \mathrm{L}$ pipette tip. Phase images and cell areas at several time points after scratch were calculated using MiToBo software as previously described [51].

\section{Immunoblotting}

Immunoblot analysis was carried out using the NuPAGE manufacture's protocol. The primary antibodies used in this study include: Notch1 (3608; Cell Signaling), cleaved Notch1 (4147; Cell Signaling) anti-phospho-p44/ p42 MAP kinase (4370; Cell Signaling), anti-p44/p42 MAP kinase (9102; Cell Signaling), anti-phospho AKT (9271; Cell Signaling), anti-AKT (9272; Cell Signaling), anti-MEK1/2 (8727; Cell Signaling), and anti-phosphoMEK1/2 (9154; Cell Signaling). Membranes were washed and incubated with horseradish peroxidase-conjugated secondary antibodies.

\section{IC 50 assays}

$3 \times 10^{3}$ cells were seeded in triplicate on day 0 and exposed to serial dilutions of indicated drug in media containing $0.2 \mathrm{ng} / \mathrm{mL}$ of EGF on day 1. AlamarBlue (Life Technologies) was used to determine cell proliferation according to the manufacturer's protocol. On day 6 of assay, cells were counted using a Beckman Coulter counter and percent viability was calculated using the average cell count for each variant normalized to the appropriate DMSO control. IC50 values were calculated using the $\log$ (inhibitor) vs. response - variable slope (four parameters) nonlinear regression function in Graphpad Prism 5. All cell lines were counted in triplicate. IC50s for each variant were graphed as a function of indicated drug concentration. 


\section{Microarray}

MCF10A A2441T and TWT were propagated under normal growth conditions before RNA isolation. Gene expression profiling was performed using the Affimetrix Clariom $^{\mathrm{TM}}$ D human Assay (ThermoFisher scientific), processed on the GeneChip ${ }^{\mathrm{TM}} 3000$ by the JHMI microarray core service. Relative gene expression was determined using the log fold change (LogFC) of mutants compared to parental controls. Analyses with corresponding figures were generated using the Transcriptome Analysis Console (TAC) software (ThermoFisher scientific) specific for Clariom D assay. Classification of cancer-associated genes were confirmed by identification in previous literature [40].

\section{METABRIC data analysis}

Analysis of the METABRIC dataset was used to compare overall survival (OS) and progression free survival (PFS) of patients with and without NOTCH1 variants when treated with chemotherapy. Within the METABRIC dataset $(n=2509)$, patients with ER-/ HER- $(n=309)$ were selected from the 3-gene classifier and those who received chemotherapy $(n=149)$ were analyzed. Among these patients, 11 patients had at least 1 NOTCH1 variant and 138 patients were classified as WT. Statistical analysis and Kaplan-Meier curves were generated by the cBioPortal algorithm. To obtain the NOTCH1 dataset, select Breast $>$ Breast Cancer (METABRIC, Nature 2012 \& Nat Commun 2016) ( $n$ $=2509)>3$ Gene Classifier Subtypes: ER-/HER- $(n=$ 309) > Chemotherapy: Yes $(n=149)>$ Mutated Genes: NOTCH1 $(n=11)$. To obtain the WT dataset utilize the custom selection to remove the NOTCH1 variants and sort for ER-/HER2- and Chemotherapy status.

\section{Statistics}

All statistical analyses were performed using GraphPad Prism software. 2-way ANOVA tests were used to compare the experimental groups to the corresponding controls. Significance levels are indicated by one or more asterisk: ${ }^{*} p \leq 0.05,{ }^{* *} p \leq 0.01$ and ${ }^{* * *} p \leq 0.001$. Error bars represent \pm SEM.

\section{Author contributions}

B.H.P and K.C. conceived the project. B.H.P and S.C supervised the project. K.C., M.P, and D.H.S. developed protocols and designed experiments. K.C., M.P., D.S., R.B., D.C., D.J.Z., S.K., I.G.W., N.H., D.M.R., K.K.S., W.B.D., B.B., D.S., and H.Y.W. carried out experiments and experimental analysis, K.C., M.P., D.H.S., S.C., and B.H.P analyzed and interpreted data. J.D, H.Y.W., and P.J.H. contributed to data interpretation and troubleshooting discussions. K.C., D.H., M.P., S.C., and B.H.P wrote the manuscript with comments from all co-authors.

\section{CONFLICTS OF INTEREST}

B.H.P. is a paid consultant for Jackson Labs, EQRx, Sermonix, Hologics, Guardant Health and is a paid scientific advisory board member for Celcuity Inc. B.H.P. also has research contracts with GE Healthcare, Lilly and Pfizer. Under separate licensing agreements between Horizon Discovery, LTD and The Johns Hopkins University, B.H.P. is entitled to a share of royalties received by the University on sales of products. The terms of this arrangement are being managed by the Johns Hopkins University in accordance with its conflict of interest policies. J.D. receives royalties from patents held by City of Hope and Thomas Jefferson University. J.D. also holds stock awards from Xilio Therapeutics. None of these listed had do direct conflict of interest on the project.

\section{FUNDING}

This work was supported by: American Cancer Society 131356-RSG-17-160-01-CSM (PJH); National Cancer Institute/National Institute of Health RO1CA211695-01A1(PJH); James Rowen (PJH); VUMC MSTP Program NIH T32GM007347 (RB); B.H.P. is supported by The Breast Cancer Research Foundation, Komen Foundation, and NIH CA214494/CA194024 (B.H.P.). We would also like to thank and acknowledge the support of The Canney Foundation, the Marcie and Ellen Foundation, Donna and John Hall, The Cornelius A. Craig Chair, Steve Kandell, Amy and Barry Baker and the Vanderbilt-Ingram Cancer Center support grant (NIH CA068485) and Breast Cancer SPORE (NIH CA098131).

\section{REFERENCES}

1. Lehmann BD, Bauer JA, Chen X, Sanders ME, Chakravarthy AB, Shyr Y, Pietenpol JA. Identification of human triple-negative breast cancer subtypes and preclinical models for selection of targeted therapies. J Clin Invest. 2011; 121:2750-67. https://doi.org/10.1172/ JCI45014. [PubMed]

2. Park JH, Ahn JH, Kim SB. How shall we treat early triple-negative breast cancer (TNBC): from the current standard to upcoming immuno-molecular strategies. ESMO Open. 2018; 3:e000357. https://doi.org/10.1136/ esmoopen-2018-000357. [PubMed]

3. Collins LC, Marotti JD, Gelber S, Cole K, Ruddy K, Kereakoglow S, Brachtel EF, Schapira L, Come SE, Winer EP, Partridge AH. Pathologic features and molecular phenotype by patient age in a large cohort of young women with breast cancer. Breast Cancer Res Treat. 2012; 
131:1061-66. https://doi.org/10.1007/s10549-011-1872-9. [PubMed]

4. Wen S, Manuel L, Doolan M, Westhuyzen J, Shakespeare TP, Aherne NJ. Effect of Clinical and Treatment Factors on Survival Outcomes of Triple Negative Breast Cancer Patients. Breast Cancer (Dove Med Press). 2020; 12:27-35. https://doi.org/10.2147/BCTT.S236483. [PubMed]

5. Lee A, Djamgoz MBA. Triple negative breast cancer: Emerging therapeutic modalities and novel combination therapies. Cancer Treat Rev. 2018; 62:110-22. https://doi. org/10.1016/j.ctrv.2017.11.003. [PubMed]

6. Santagata S, Demichelis F, Riva A, Varambally S, Hofer MD, Kutok JL, Kim R, Tang J, Montie JE, Chinnaiyan AM, Rubin MA, Aster JC. JAGGED1 expression is associated with prostate cancer metastasis and recurrence. Cancer Res. 2004; 64:6854-57. https://doi.org/10.1158/0008-5472. CAN-04-2500. [PubMed]

7. Hassan WA, Yoshida R, Kudoh S, Hasegawa K, NiimoriKita K, Ito T. Notch1 controls cell invasion and metastasis in small cell lung carcinoma cell lines. Lung Cancer. 2014; 86:304-10. https://doi.org/10.1016/j.lungcan.2014.10.007. [PubMed]

8. Shao S, Zhao X, Zhang X, Luo M, Zuo X, Huang S, Wang Y, Gu S, Zhao X. Notch1 signaling regulates the epithelialmesenchymal transition and invasion of breast cancer in a Slug-dependent manner. Mol Cancer. 2015; 14:28. https:// doi.org/10.1186/s12943-015-0295-3. [PubMed]

9. Lin J, Xu Z, Xie J, Deng X, Jiang L, Chen H, Peng C, Li H, Zhang J, Shen B. Oncogene APOL1 promotes proliferation and inhibits apoptosis via activating NOTCH1 signaling pathway in pancreatic cancer. Cell Death Dis. 2021; 12:760. https://doi.org/10.1038/s41419-021-03985-1. [PubMed]

10. Bin Hafeez B, Adhami VM, Asim M, Siddiqui IA, Bhat KM, Zhong W, Saleem M, Din M, Setaluri V, Mukhtar H. Targeted knockdown of Notch1 inhibits invasion of human prostate cancer cells concomitant with inhibition of matrix metalloproteinase-9 and urokinase plasminogen activator. Clin Cancer Res. 2009; 15:452-59. https://doi. org/10.1158/1078-0432.CCR-08-1631. [PubMed]

11. van Es JH, van Gijn ME, Riccio O, van den Born M, Vooijs M, Begthel H, Cozijnsen M, Robine S, Winton DJ, Radtke F, Clevers H. Notch/gamma-secretase inhibition turns proliferative cells in intestinal crypts and adenomas into goblet cells. Nature. 2005; 435:959-63. https://doi. org/10.1038/nature03659. [PubMed]

12. Weng AP, Ferrando AA, Lee W, Morris JP 4th, Silverman LB, Sanchez-Irizarry C, Blacklow SC, Look AT, Aster JC. Activating mutations of NOTCH1 in human T cell acute lymphoblastic leukemia. Science. 2004; 306:269-71. https://doi.org/10.1126/science.1102160. [PubMed]

13. Westhoff B, Colaluca IN, D'Ario G, Donzelli M, Tosoni D, Volorio S, Pelosi G, Spaggiari L, Mazzarol G, Viale G, Pece S, Di Fiore PP. Alterations of the Notch pathway in lung cancer. Proc Natl Acad Sci U S A. 2009; 106:22293-98. https://doi.org/10.1073/pnas.0907781106. [PubMed]
14. Guo S, Liu M, Gonzalez-Perez RR. Role of Notch and its oncogenic signaling crosstalk in breast cancer. Biochim Biophys Acta. 2011; 1815:197-213. https://doi. org/10.1016/i.bbcan.2010.12.002. [PubMed]

15. Wei P, Walls M, Qiu M, Ding R, Denlinger RH, Wong A, Tsaparikos K, Jani JP, Hosea N, Sands M, Randolph S, Smeal T. Evaluation of selective gamma-secretase inhibitor PF-03084014 for its antitumor efficacy and gastrointestinal safety to guide optimal clinical trial design. Mol Cancer Ther. 2010; 9:1618-28. https://doi.org/10.1158/1535-7163. MCT-10-0034. [PubMed]

16. Roy M, Pear WS, Aster JC. The multifaceted role of Notch in cancer. Curr Opin Genet Dev. 2007; 17:52-59. https:// doi.org/10.1016/i.gde.2006.12.001. [PubMed]

17. Carrieri FA, Dale JK. Turn It Down a Notch. Front Cell Dev Biol. 2016; 4:151. https://doi.org/10.3389/fcell.2016.00151. [PubMed]

18. Wang K, Zhang Q, Li D, Ching K, Zhang C, Zheng X, Ozeck M, Shi S, Li X, Wang H, Rejto P, Christensen J, Olson P. PEST domain mutations in Notch receptors comprise an oncogenic driver segment in triple-negative breast cancer sensitive to a $\gamma$-secretase inhibitor. Clin Cancer Res. 2015; 21:1487-96. https://doi.org/10.1158/1078-0432.CCR-14-1348. [PubMed]

19. Callahan R, Egan SE. Notch signaling in mammary development and oncogenesis. J Mammary Gland Biol Neoplasia. 2004; 9:145-63. https://doi.org/10.1023/ B:JOMG.0000037159.63644.81. [PubMed]

20. Bhanushali AA, Babu S, Thangapandi VR, Pillai R, Chheda $\mathrm{P}$, Das BR. Mutations in the HD and PEST domain of Notch-1 receptor in T-cell acute lymphoblastic leukemia: report of novel mutations from Indian population. Oncol Res. 2010; 19:99-104. https://doi.org/10.3727/09650401 0x12864748215007. [PubMed]

21. Puente XS, Pinyol M, Quesada V, Conde L, Ordóñez GR, Villamor N, Escaramis G, Jares P, Beà S, González-Díaz M, Bassaganyas L, Baumann T, Juan M, et al. Whole-genome sequencing identifies recurrent mutations in chronic lymphocytic leukaemia. Nature. 2011; 475:101-5. https:// doi.org/10.1038/nature10113. [PubMed]

22. Giuli MV, Giuliani E, Screpanti I, Bellavia D, Checquolo S. Notch Signaling Activation as a Hallmark for Triple-Negative Breast Cancer Subtype. J Oncol. 2019; 2019:8707053. https://doi.org/10.1155/2019/8707053. [PubMed]

23. Piwarski SA, Thompson C, Chaudhry AR, Denvir J, Primerano DA, Fan J, Salisbury TB. The putative endogenous AHR ligand ITE reduces JAG1 and associated NOTCH1 signaling in triple negative breast cancer cells. Biochem Pharmacol. 2020; 174:113845. https://doi. org/10.1016/j.bcp.2020.113845. [PubMed]

24. Cerami E, Gao J, Dogrusoz U, Gross BE, Sumer SO, Aksoy BA, Jacobsen A, Byrne CJ, Heuer ML, Larsson E, Antipin Y, Reva B, Goldberg AP, et al. The cBio cancer genomics portal: an open platform for exploring multidimensional cancer genomics data. Cancer Discov. 2012; 2:401-4. https://doi.org/10.1158/2159-8290.CD-12-0095. [ubMed] 
25. Reedijk M, Odorcic S, Chang L, Zhang H, Miller N, McCready DR, Lockwood G, Egan SE. High-level coexpression of JAG1 and NOTCH1 is observed in human breast cancer and is associated with poor overall survival. Cancer Res. 2005; 65:8530-37. [PubMed]

26. Lee SM, Moon J, Redman BG, Chidiac T, Flaherty LE, Zha Y, Othus M, Ribas A, Sondak VK, Gajewski TF, Margolin KA. Phase 2 study of RO4929097, a gamma-secretase inhibitor, in metastatic melanoma: SWOG 0933. Cancer. 2015; 121:432-40. https://doi.org/10.1002/cncr.29055. [PubMed]

27. Massard C, Azaro A, Soria JC, Lassen U, Le Tourneau C, Sarker D, Smith C, Ohnmacht U, Oakley G, Patel BKR, Yuen ESM, Benhadji KA, Rodon J. First-in-human study of LY3039478, an oral Notch signaling inhibitor in advanced or metastatic cancer. Ann Oncol. 2018; 29:1911-17. https:// doi.org/10.1093/annonc/mdy244. [PubMed]

28. Sardesai S, Badawi M, Mrozek E, Morgan E, Phelps M, Stephens J, Wei L, Kassem M, Ling Y, Lustberg M, Stover D, Williams N, Layman R, et al. A phase I study of an oral selective gamma secretase (GS) inhibitor RO4929097 in combination with neoadjuvant paclitaxel and carboplatin in triple negative breast cancer. Invest New Drugs. 2020; 38:1400-10. https://doi.org/10.1007/s10637-020-00895-5. [PubMed]

29. Zhao M, Kim P, Mitra R, Zhao J, Zhao Z. TSGene 2.0: an updated literature-based knowledgebase for tumor suppressor genes. Nucleic Acids Res. 2016; 44:D1023-31. https://doi.org/10.1093/nar/gkv1268. [PubMed]

30. Tate JG, Bamford S, Jubb HC, Sondka Z, Beare DM, Bindal N, Boutselakis H, Cole CG, Creatore C, Dawson E, Fish P, Harsha B, Hathaway C, et al. COSMIC: the Catalogue Of Somatic Mutations In Cancer. Nucleic Acids Res. 2019; 47:D941-47. https://doi.org/10.1093/nar/gky1015. [PubMed]

31. Hanahan D, Weinberg RA. Hallmarks of cancer: the next generation. Cell. 2011; 144:646-74. https://doi. org/10.1016/j.cell.2011.02.013. [PubMed]

32. Ignatoski KM, Lapointe AJ, Radany EH, Ethier SP. erbB-2 overexpression in human mammary epithelial cells confers growth factor independence. Endocrinology. 1999; 140:361522. https://doi.org/10.1210/endo.140.8.6939. [PubMed]

33. De Luca A, Maiello MR, D'Alessio A, Pergameno M, Normanno N. The RAS/RAF/MEK/ERK and the PI3K/ AKT signalling pathways: role in cancer pathogenesis and implications for therapeutic approaches. Expert Opin Ther Targets. 2012; 16:S17-27. https://doi.org/10.1517/1472822 2.2011.639361. [PubMed]

34. Shin SI, Freedman VH, Risser R, Pollack R. Tumorigenicity of virus-transformed cells in nude mice is correlated specifically with anchorage independent growth in vitro. Proc Natl Acad Sci U S A. 1975; 72:4435-39. https://doi. org/10.1073/pnas.72.11.4435. [PubMed]

35. Konishi H, Karakas B, Abukhdeir AM, Lauring J, Gustin JP, Garay JP, Konishi Y, Gallmeier E, Bachman KE, Park $\mathrm{BH}$. Knock-in of mutant K-ras in nontumorigenic human epithelial cells as a new model for studying K-ras mediated transformation. Cancer Res. 2007; 67:8460-67. https://doi. org/10.1158/0008-5472.CAN-07-0108. [PubMed]

36. Wang GM, Wong HY, Konishi H, Blair BG, Abukhdeir AM, Gustin JP, Rosen DM, Denmeade SR, Rasheed Z, Matsui W, Garay JP, Mohseni M, Higgins MJ, et al. Single copies of mutant KRAS and mutant PIK3CA cooperate in immortalized human epithelial cells to induce tumor formation. Cancer Res. 2013; 73:3248-61. https://doi. org/10.1158/0008-5472.CAN-12-1578. [PubMed]

37. Debnath J, Muthuswamy SK, Brugge JS. Morphogenesis and oncogenesis of MCF-10A mammary epithelial acini grown in three-dimensional basement membrane cultures. Methods. 2003; 30:256-68. https://doi.org/10.1016/s10462023(03)00032-x. [PubMed]

38. Bellon M, Moles R, Chaib-Mezrag H, Pancewicz J, Nicot C. JAG1 overexpression contributes to Notch1 signaling and the migration of HTLV-1-transformed ATL cells. J Hematol Oncol. 2018; 11:119. https://doi.org/10.1186/s13045-0180665-6. [PubMed]

39. Zhou Q, Wang Y, Peng B, Liang L, Li J. The roles of Notch1 expression in the migration of intrahepatic cholangiocarcinoma. BMC Cancer. 2013; 13:244. https:// doi.org/10.1186/1471-2407-13-244. [PubMed]

40. Cancer Genome Atlas Network. Comprehensive molecular portraits of human breast tumours. Nature. 2012; 490:6170. https://doi.org/10.1038/nature11412. [PubMed]

41. Wang Q, Xu M, Sun Y, Chen J, Chen C, Qian C, Chen Y, Cao L, Xu Q, Du X, Yang W. Gene Expression Profiling for Diagnosis of Triple-Negative Breast Cancer: A Multicenter, Retrospective Cohort Study. Front Oncol. 2019; 9:354. https://doi.org/10.3389/fonc.2019.00354. [PubMed]

42. Stoeck A, Lejnine S, Truong A, Pan L, Wang H, Zang C, Yuan J, Ware C, MacLean J, Garrett-Engele PW, Kluk M, Laskey J, Haines BB, et al. Discovery of biomarkers predictive of GSI response in triple-negative breast cancer and adenoid cystic carcinoma. Cancer Discov. 2014; 4:1154-67. https://doi.org/10.1158/2159-8290.CD-13-0830. [PubMed]

43. Robinson DR, Kalyana-Sundaram S, Wu YM, Shankar S, Cao X, Ateeq B, Asangani IA, Iyer M, Maher CA, Grasso CS, Lonigro RJ, Quist M, Siddiqui J, et al. Functionally recurrent rearrangements of the MAST kinase and Notch gene families in breast cancer. Nat Med. 2011; 17:1646-51. https://doi.org/10.1038/nm.2580. [PubMed]

44. Locatelli MA, Aftimos P, Dees EC, LoRusso PM, Pegram MD, Awada A, Huang B, Cesari R, Jiang Y, Shaik MN, Kern KA, Curigliano G. Phase I study of the gamma secretase inhibitor PF-03084014 in combination with docetaxel in patients with advanced triple-negative breast cancer. Oncotarget. 2017; 8:2320-28. https://doi.org/10.18632/ oncotarget.13727. [PubMed]

45. Zhang CC, Pavlicek A, Zhang Q, Lira ME, Painter CL, Yan Z, Zheng X, Lee NV, Ozeck M, Qiu M, Zong Q, Lappin PB, Wong A, et al. Biomarker and pharmacologic evaluation 
of the $\gamma$-secretase inhibitor PF-03084014 in breast cancer models. Clin Cancer Res. 2012; 18:5008-19. https://doi. org/10.1158/1078-0432.CCR-12-1379. [PubMed]

46. Yuan X, Zhang M, Wu H, Xu H, Han N, Chu Q, Yu S, Chen Y, Wu K. Expression of Notch1 Correlates with Breast Cancer Progression and Prognosis. PLoS One. 2015; 10:e0131689. https://doi.org/10.1371/journal. pone.0131689. [ [PubMed]

47. Kumar B, Prasad M, Bhat-Nakshatri P, Anjanappa M, Kalra M, Marino N, Storniolo AM, Rao X, Liu S, Wan J, Liu Y, Nakshatri H. Normal Breast-Derived Epithelial Cells with Luminal and Intrinsic Subtype-Enriched Gene Expression Document Interindividual Differences in Their Differentiation Cascade. Cancer Res. 2018; 78:510723. https://doi.org/10.1158/0008-5472.CAN-18-0509. [PubMed]

48. Kyker-Snowman K, Hughes RM, Yankaskas CL, Cravero K, Karthikeyan S, Button B, Waters I, Rosen DM, Dennison L, Hunter N, Donaldson J, Christenson ES, Konstantopoulos $\mathrm{K}$, et al. TrkA overexpression in nontumorigenic human breast cell lines confers oncogenic and metastatic properties. Breast Cancer Res Treat. 2020; 179:631-42. https://doi.org/10.1007/s10549-019-05506-3. [PubMed]

49. Mollen EWJ, Ient J, Tjan-Heijnen VCG, Boersma LJ, Miele L, Smidt ML, Vooijs MAGG. Moving Breast Cancer Therapy up a Notch. Front Oncol. 2018; 8:518. https://doi. org/10.3389/fonc.2018.00518. [PubMed]

50. Domingo-Domenech J, Vidal SJ, Rodriguez-Bravo V, Castillo-Martin M, Quinn SA, Rodriguez-Barrueco R, Bonal DM, Charytonowicz E, Gladoun N, de la Iglesia-Vicente J, Petrylak DP, Benson MC, Silva JM, Cordon-Cardo C.
Suppression of acquired docetaxel resistance in prostate cancer through depletion of notch- and hedgehog-dependent tumor-initiating cells. Cancer Cell. 2012; 22:373-88. https:// doi.org/10.1016/j.ccr.2012.07.016. [PubMed]

51. Zabransky DJ, Yankaskas CL, Cochran RL, Wong HY, Croessmann S, Chu D, Kavuri SM, Red Brewer M, Rosen DM, Dalton WB, Cimino-Mathews A, Cravero K, Button $B$, et al. HER2 missense mutations have distinct effects on oncogenic signaling and migration. Proc Natl Acad Sci U S A. 2015; 112:E6205-14. https://doi.org/10.1073/ pnas.1516853112. [PubMed]

52. Gustin JP, Karakas B, Weiss MB, Abukhdeir AM, Lauring J, Garay JP, Cosgrove D, Tamaki A, Konishi H, Konishi Y, Mohseni M, Wang G, Rosen DM, et al. Knockin of mutant PIK3CA activates multiple oncogenic pathways. Proc Natl Acad Sci U S A. 2009; 106:2835-40. https://doi. org/10.1073/pnas.0813351106. [PubMed]

53. Konishi H, Lauring J, Garay JP, Karakas B, Abukhdeir AM, Gustin JP, Konishi Y, Park BH. A PCR-based highthroughput screen with multiround sample pooling: application to somatic cell gene targeting. Nat Protoc. 2007; 2:2865-74. https://doi.org/10.1038/nprot.2007.409. [PubMed]

54. Croessmann S, Wong HY, Zabransky DJ, Chu D, Rosen DM, Cidado J, Cochran RL, Dalton WB, Erlanger B, Cravero K, Button B, Kyker-Snowman K, Hurley PJ, et al. PIK3CA mutations and TP53 alterations cooperate to increase cancerous phenotypes and tumor heterogeneity. Breast Cancer Res Treat. 2017; 162:451-64. https://doi. org/10.1007/s10549-017-4147-2. [PubMed] 